\title{
2014 HUNCH SPIDER
}

\author{
Jonathon Woods
}

East Troy High School NASA HUNCH

\section{Program}

HUNCH is an instructional partnership between NASA and high school, intermediate school, and middle school students. This partnership benefits both NASA and students. NASA receives cost-effective hardware, while students receive real-world hands-on experiences. The results of this cooperation is the inspiration for the next generation of researchers and space explorers. Our team was comprised of seven members: Eli Rand, Nicholas Nolting, Jonathon Woods, Luke Goetsch, Paul D'Amico, Joey Dominski, and Daniel Barr. Each member of our team had designated roles within the group, but we all worked together to advance towards goals and meet deadlines.

\section{Goal}

The task at hand was to design, and build an experiment to test in microgravity. As with each team coming up with their own unique experiment, our team went with trying to solve the problem of astronauts always losing tools that they are working with aboard the International Space Station. This has always been a challenging issue for astronauts in space due to the microgravity environment. This problem is a potential high risk due to when tools or parts float away as they can get lost, lodged somewhere, or even damage other equipment. By creation of our device we hope to improve the livability and ensure greater safety for astronauts in space.

\section{Experiment}

Our team went with the route of using RFID (Radio Frequency Identification) technology as the way to alert a user about an object leaving their vicinity. The experiment SPIDER (Spatial Proximity Identification Device for Environmental Reading) consisted of several parts.

SPIDER's was made of an Arduino board, RFID board, Nano rack, antenna, and tag. These parts were for controlling/interpreting data, reading, housing the device, transmitting the signal/detecting the tag, and marking the tool, respectively. The program for SPIDER is on a continuous loop, searching for a signal from the RFID tag within a range of 3 feet (average arm reach). If the signal is lost it causes a blue light to flash at the user, alerting them of the objects movement. 


\section{Diagram}

Antenna (902-928Mhz)

RFID Module (Interprets Data)

LEDs (Indicate in/out of range) Arduino Board (Utilizes Data)

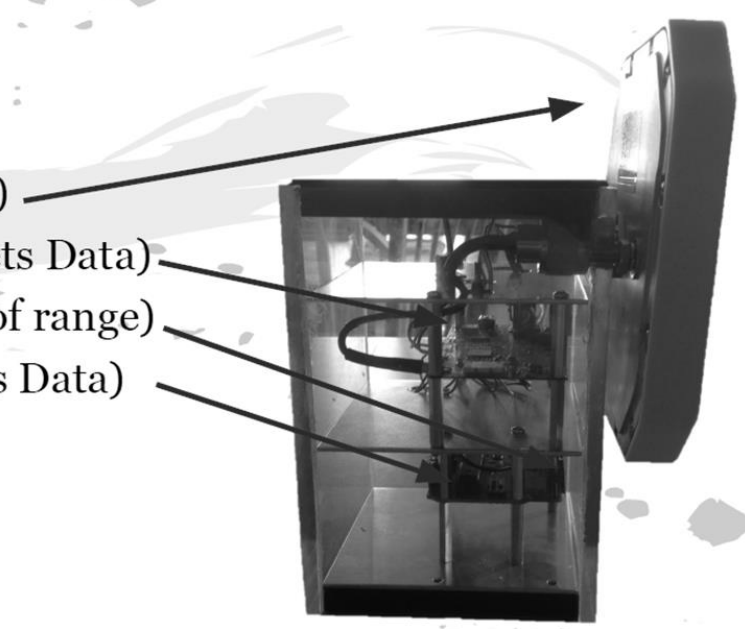

\section{Testing}

Testing and data collection was done in two parts. The first part, ground testing, was done to ensure all parts of the experiment were working properly with one another, and that all bugs had been worked out of the program. This was done weekly over the course of three months before flight testing. Ground testing involved moving the RFID tag away from the antenna to find the best distance to signal ratio for the human arm reach and tweaking the program for consistency. The second part of testing was done during two reduced gravity flights. Each flight consisted of 30 parabolas, lasting between 30-15 seconds of micro-gravity. Each one of our tests was done during the 30-15 seconds of reduced gravity to procure the best results. Testing on the first flight involved moving the RFID tag at various angles within a NASA tested safety glove box. These angles were pre-measured out at $45^{\circ}, 67^{\circ}$, and $90^{\circ}$ from the antenna. The angles were tested because the antenna can only send and receive signals from items on a certain axis. We wanted to see how the angles would make the signal deteriorate as the tag was placed farther away from the axis directly perpendicular to the antenna face. Testing on flight two was made up of letting the tag float freely outside the glove box, and marking when the signal with the device was lost. The device was tested in free fall because we wanted to make sure it would be unaffected and to measure any affects that could have possibly arisen. There was no research done to test RFID in microgravity and we did not want to assume. All of this testing was done, on the reduced gravity flight, to ensure the reliability and effectiveness of the devices NASA could potentially put op on the ISS. 


\section{Outcome}

During both flights the testing was congruent with ground testing. The first experiment gave us consistent data that from $90^{\circ}$ to $45^{\circ}$ the average distance decreased by $30 \%$. This means that our current model is only practical if the tag is within about a $45^{\circ}$ angle from the axis perpendicular to the face of the antenna. Staying constant with how SPIDER was programmed, the device lost signal with the tag within the 3 foot radius when at a $90^{\circ}$ angle to the antenna. In the future we're planning on furthering our use with RFID technology. We have many ideas about the next steps we want to take with the project; these include but are not limited to: an omnidirectional antenna to have a wider area for detection, several antennas for detecting location or direction of travel, and lastly fractal antennas which give you more powerful antennas within the same space. 